\title{
ESTUDIOS QUIMIOTAXONOMICOS DE BURSERA (BURSERACEAE). II. UNA ESPECIE NUEVA DE ORIGEN HIBRIDO DE LA BARRANCA DE TOLANTONGO, ESTADO DE HIDALGO 1
}

\author{
JERZY RZEDOWSKI \\ Instituto de Ecología, Centro Regional del Bajío \\ Apartado postal 386; 61600 Pátzcuaro, Mich. México \\ Evangelina Ortiz \\ Escuela Nacional de Ciencias Biológicas \\ Instituto Politécnico Nacional \\ 11340 México, D.F.
}

\section{RESUMEN}

Se describe como nueva a Bursera medranoana, especie que, tanto por sus características morfológicas como por la composición de sus terpenoides, muestra ser de origen híbrido a partir de $B$. morelensis Ramirez y B. schlechtendalii Engl. Las tres especies conviven en el fondo de la Barranca de Tolantongo, al E de Ixmiquilpan, Hidalgo (México), donde B. morelensis forma bosquecillos en algunas laderas de fuerte pendiente. En ciertos sitios, sin embargo, la densidad de individuos de B. medranoana iguala a la de los correspondientes de B. morelensis.

\section{ABSTRACT}

Bursera medranoana sp.n. originated as a hybrid between B. morelensis Ramírez and $B$. schlechtendalii Engl., as can be proved by its morphological characters and its terpenoid composition. The three species occur together at the bottom of the Barranca de Tolantongo, east of Ixmiquilpan (state of Hidalgo, Mexico), where B. morelensis forms low forests on steep slopes. In a few localities, however, the density of trees of $B$. medranoana equals that of $B$. morelensis.

Dentro del sistema de profundos cañones que han excavado los afluentes del río Pánuco en la Sierra Madre Oriental, la Barranca de Tolantongo es una de las más espectaculares y también de las más interesantes desde el punto de vista botánico. Sus abruptas laderas, en su mayor parte, están cubiertas por varios tipos de matorrales xerófilos (Hiriart Valencia, 1981), pero en algunas porciones cercanas al fondo, sobre todo en áreas de exposición sur o de otra manera protegidas, existen manchones de bosquete de Bursera morelensis Ramírez, árbol conspicuo por la coloración rojiza de su tronco.

La presencia de tales comunidades vegetales se conocía ya desde hace algún tiempo, no solamente de este lugar, sino practicamente de todos los cañones del sistema fluvial mencionado, desde Hidalgo hasta San Luis Potosí, pero una exploración más cuidadosa de la Barranca de Tolantongo llevó al hallazgo de otras plantas del mismo género dentro de uno de

\footnotetext{
- Trabajo realizado con apoyo del Consejo Nacional de Ciencia y Tecnología y del Centro de Investigación y Desarrollo del Estado de Michoacán.
} 
los bosquetes mencionados. No muy lejos de la zona turística, ligada a las grutas, se colectaron también muestras de B. schlechtendaliiEngl. así como de un arbolito con hojas parecidas a las de $B$. fagaroides (HBK.) Engl., pero con corteza rojiza y aroma muy fuerte, análogo al de $B$. morelensis.

De inmediato se consideró que este último podría representar un híbrido entre $B$. morelensis y $B$. schlechtendalii y como tal se identificaron de manera tentativa los primeros ejemplares. Posteriormente se pensó en la conveniencia de muestrear todas las plantas involucradas para realizar análisis cromatográfico comparativo de sus terpenoides, lo cual pudo llevarse a cabo con algunas dificultades, puesto que la fructificación en ese año particular fue escasa y finalmente se optó por extraer el aceite de la corteza.

Al estar realizando la búsqueda de plantas confruto, se pudieron, sin embargo, descubrir varios fenómenos interesantes: 1.- En algunas laderas de la Barranca los individuos del supuesto híbrido (de ahora en adelante denominado $B$. medranoana) son tan abundantes como los de $B$. morelensis y mucho más comunes que los de $B$. schlechtendalii (relación aproximada de densidad de $15: 15: 1$ ). 2.- En $B$. medranoana son frecuentes las irregularidades en la forma de las hojas, afectando sobre todo al foliolo terminal, pero a menudo también a los foliolos laterales. 3.- Todos los arbolitos de $B$. medranoana producen frutos, aunque con frecuencia no en grandes cantidades. 4.- No se localizó ninguna planta con características intermedias entre $B$. medranoana y $B$. morelensis, aunque se encontraron cinco que podrían interpretarse como resultado de retrocruzamiento entre la primera y $B$. schlechtendalii, mismas que también se muestrearon para análisis químico.

Las observaciones 2 y 3 , desde luego, constituyen un contundente apoyo a la hipótesis de que $B$. medranoana es una población de origen híbrido.

Posteriormente, al visitar la localidad en la época de floración, se pudo comprobar que todos los individuos de $B$. medranoana llevan un solo tipo de flores, que son morfológicamente hermafroditas, pero funcionalmente femeninas, pues en ninguna de las anteras examinadas se encontró huella de polen.

En el cuadro 1 se comparan algunos caracteres de B. morelensis, B. schlechtendalii, $B$. medranoana, así como de $B$. aff. simaruba (L.) Sarg., otra especie que prospera a la orilla del río de la Barranca, a veces en lugares cercanos a los bosquetes de $B$. morelensis. Todas estas plantas presentan corteza rojiza exfoliante y tienen frutos trivalvados, por lo cual pertenecen a la sección típica (sect. Bursera) del género.

De la información que ofrece el cuadro cabe deducir que, si bien los caracteres de $B$. medranoana en muchos casos son intermedios entre $B$. morelensis y $B$. schlechtendalii y en otros coinciden con alguno de estos posibles progenitores, de ninguna manera debe descartarse la posibilidad de que $B$. aff. simaruba haya intervenido en el cruzamiento. Sobre todo, si se toma en cuenta los aspectos puramente morfológicos, la posibilidad de que se trate de un híbrido entre esta última y $B$. schlechtendalii también parece factible.

Los resultados de los análisis cromatográficos (para la metodología véase el trabajo de Rzedowski y Ortiz (1982: 74)) se resumen en el cuadro 2. En total se muestrearon 8 individuos de $B$. morelensis, 7 de $B$. schlechtendalii, 9 de $B$. medranoana, 5 del probable híbrido entre $B$. medranoana y $B$. schlechtendalii, así como 5 de B. aff. simaruba.

El aparato detectó la presencia de 25 diferentes terpenoides (componentes No. 1 a 25, ordenados por sus tiempos de retención), de los cuales varios (No. 1, 2, 3, 4, 5,7 y 8) se registraron en todas las especies y en casi todos los individuos muestreados. Aun cuando se puede observar que en general existe bastante variación intraespecífica, cada taxon tiene sus 
Rzedowski y Ortiz: Estudios Quimiotaxonómicos de Bursera

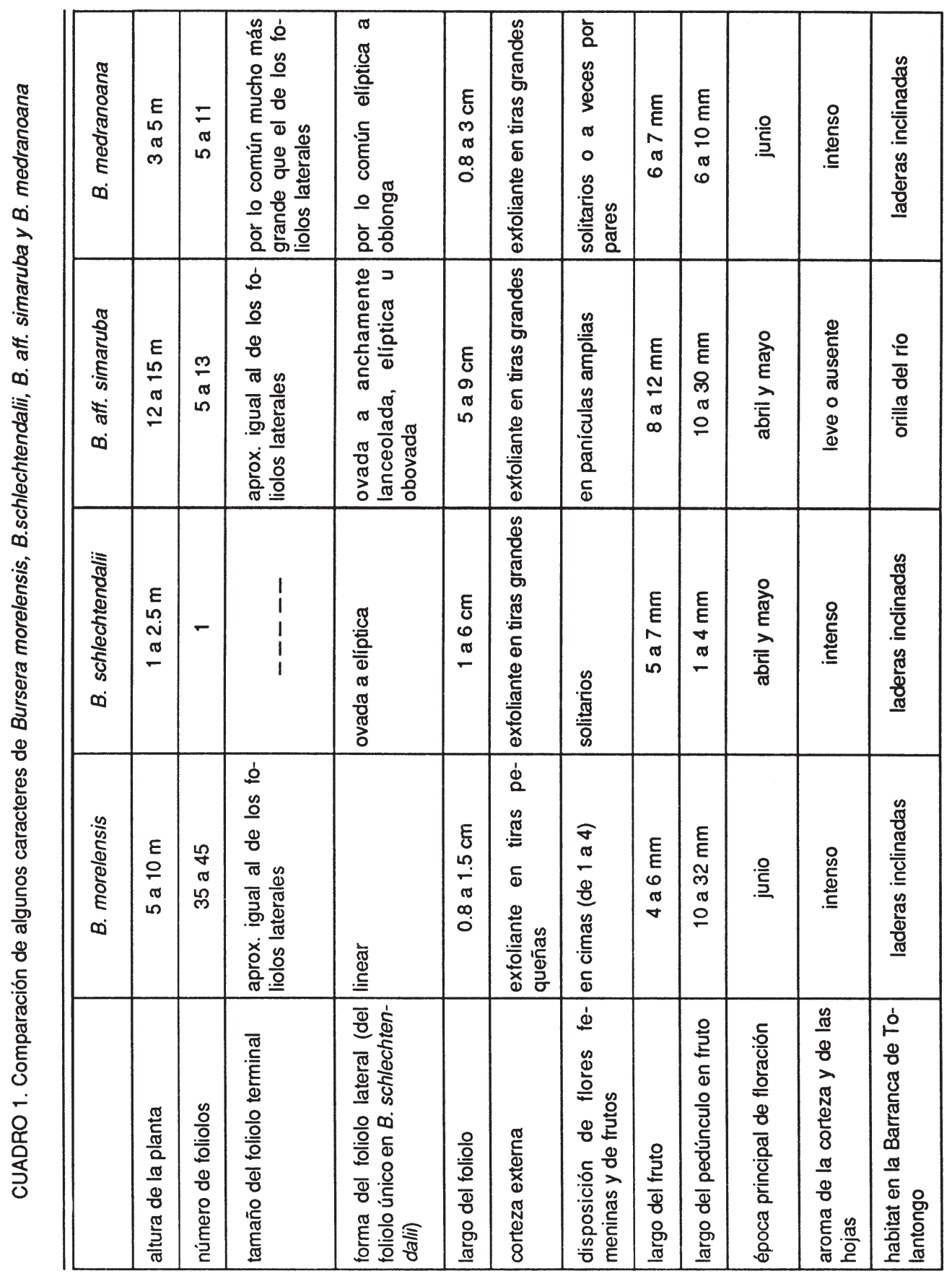




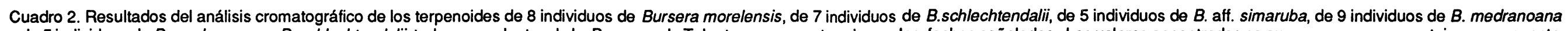

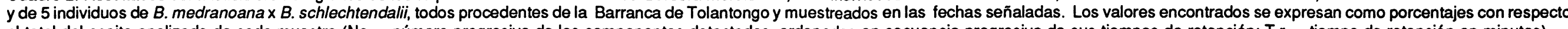

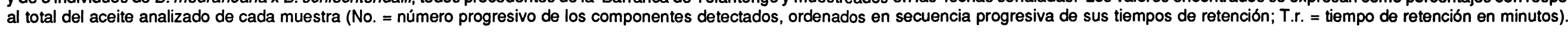

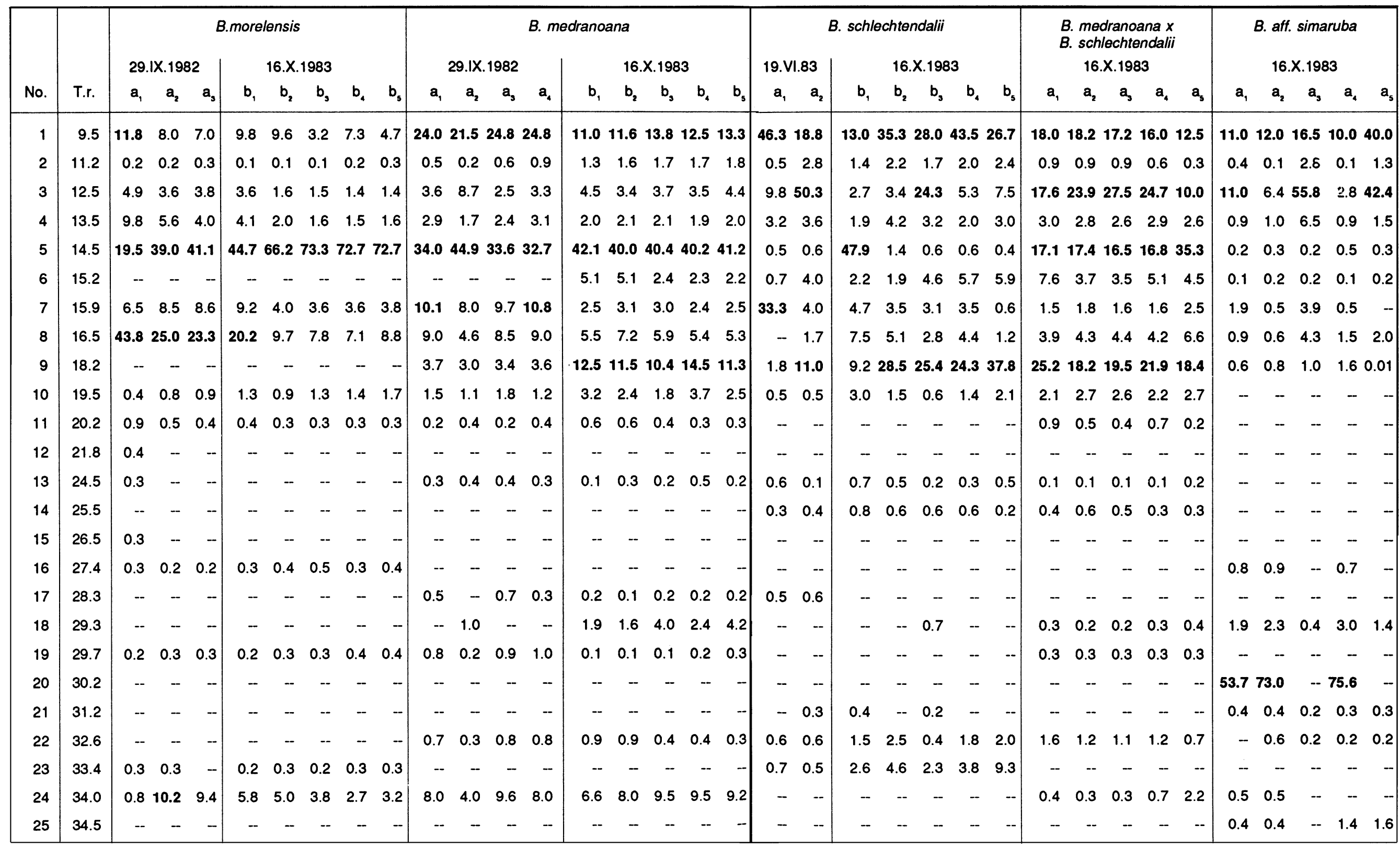


rasgos químicos definidos. Así, por ejemplo, B. morelensis presenta en forma constante valores altos en el componente 5 y a menudo también en el 8, carece de los terpenoides No. 6, 9, 14, $17,18,20,21,22$ y 25 , en cambio lleva en todos o casi todos los individuos los correspondientes a los Nos. 11 y 19 , mismos que son ausentes en $B$. schlechtendaliiy en $B$. aff. simaruba.

B. schlechtendalii, en contraste, se caracteriza por contenidos altos en el componente No. 1, a menudo asimismo en el No. 9 y con alguna frecuencia en el No. 3, carece de los terpenoides $11,12,15,16,19,20,24$ y 25 y lleva en forma constante los correspondientes a los Nos. 13 y 14, ausentes o casi ausentes en $B$. aff. simaruba y $B$. morelensis.

A su vez, $B$. aff. simaruba se asemeja a $B$. schlechtendalii en sus porcentajes constantemente altos en el componente No. 1 y con frecuencia también en el No. 3, pero a menudo los presenta muy elevados en el No. 20, que falta por completo en las dos especies de comparación, al igual que el No. 25; $B$. aff. simaruba, por otro lado, carece de los terpenoides No. $10,11,12,13,14,15,17,19$ y 23.

$B$. medranoana combina con bastante claridad las características químicas de $B$. morelensis y $B$. schlechtendalii, pues presenta valores siempre elevados tanto en el No. 5 (dominante) como en el No. 1. Con la primera tiene además en común la constancia de los terpenoides 11,19 y 24, mientras que con la segunda comparte los de los números 6, 9 (a menudo en cantidades elevadas), 13, 17 y 22. Para ser una suma "perfecta" de $B$. morelensis y B. schlechtendalii sólo le faltarían las substancias No. 14, 16 y 23.

Los híbridos entre $B$. medranoana y $B$. schlechtendalii señalan químicamente grandes semejanzas con el primero de los progenitores, pero, como era de esperarse, reflejan una intensa influencia adicional del segundo. Así, el componente No. 5 por lo general ya no es dominante, en cambio son siempre altos los valores de los Nos. 3 y 9; además aparece el del No. 14. A su vez, los porcentajes de las substancias No. 8 y 24 (características de B. morelensis) son significativamente más bajos.

Todo lo anterior indica que, en cuanto a la composición de sus terpenoides concierne, resulta también confirmada la hipótesis de que $B$. medranoana se ha originado por hibridación y que sus antecesores deben haber sido $B$. morelensis y $B$. schlechtendalii. No parece probable que tenga influencia genética de $B$. aff. simaruba, pues carece de los componentes 20 y 25 y presenta otros como los correspondientes a los Nos. 11 y 19.

El siguiente problema consiste en definir la posición taxonómica de $B$. medranoana. En virtud de lo expuesto con anterioridad, se trata muy probablemente de una especie joven, surgida en o cerca de la Barranca de Tolantongo, pues aún convive ahí con ambos progenitores, aunque tal vez en vías de eliminar a uno de ellos. Desde el punto de vista morfológico parece ser una unidad bien definida y bastante estable, ya que se han visto varios cientos de individuos, todos muy parecidos entre sí. En el aspecto reproductivo da la impresión de que las plantas no han alcanzado (¿todavía?) el nivel de sexualidad que comunmente se observa en las especies de Bursera y cabe sospechar que la apomixis juega un papel preponderante, con las consiguientes limitaciones de intercambio de material genético.

En este contexto es interesante recordar que varios híbridos interespecíficos se conocen ya en Bursera (McVaugh y Rzedowski, 1965: 322, Rzedowski, 1970: 34-35) y lo notable es que por lo general los árboles originados por cruzamiento producen flores y frutos en abundancia. Sin embargo, las observaciones del campo indican que en el caso de $B$. $x$ diversifolia Rose y en otros similares no existen sino individuos aislados con las características intermedias, casi siempre en condiciones de moderado a intenso disturbio.

En cambio, la población de B. medranoana es nutrida, al parecer ajena a la perturbación, 
vigorosa, reproductivamente bastante bien aislada y activa, por todo lo cual lo más lógico es considerarla como un taxon independiente y no como un simple híbrido. A pesar de la similitud superficial, es claro que no procedería colocarla como variedad de B. fagaroides. Aunque por lo visto conserva posibilidades de cruzamiento ocasional con $B$. schlechtendalii, tampoco resultaría recomendable ubicarla como variedad de esta última, pues las discrepancias en la morfología de la hoja son grandes. En consecuencia, se le reconoce la categoría de especie y se le describe a continuación como tal.

\section{Bursera medranoana sp. n.}

Arbuscula usque ad $5 \mathrm{~m}$ alta, cortice exteriore cupreo-rubro, papyraceo, exfolianti; folia (1-) 5-11-foliolata, foliolum terminale plerumque lateralibus valde majus, usque ad $3 \mathrm{~cm}$ longum et $1.5 \mathrm{~cm}$ latum, ovatum usque ad ellipticum, rhomboideum, oblanceolatum vel obovatum, saepe asymmetricum et irregulare, foliola lateralia usque ad $3 \mathrm{~cm}$ longa et $1 \mathrm{~cm}$ lata, plerumque elliptica usque ad oblonga, sed aeque interdum irregularia; flores masculini ignoti et ut videtur absentes; flores feminei fasciculati, solitarii vel bini, calycis lobi 3, triangulares, 1-1.2 mm longi, petala 3, oblonga, $\pm 3 \mathrm{~mm}$ longa, staminodia 6 , antheris $\pm 1 \mathrm{~mm}$ longis, ovarium triloculare, stigmata 3 ; drupae trivalvatae, subglobosae vel ovoideae, $6-7 \mathrm{~mm}$ longae, glabrae, pyrenae pseudoarillo omnino indutae.

Arbolito resinoso y con aroma intenso, hasta de $5 \mathrm{~m}$ de alto y con tronco hasta de $25 \mathrm{~cm}$ de diámetro, la copa redondeada; corteza externa del tronco y de las ramas principales rojocobriza, exfoliándose en tiras grandes, papiráceas, delgadas; ramas a menudo pruinosas, rojizas, las jóvenes corta y esparcidamente pubérulas, glabrescentes.

Hojas en su mayoría aglomeradas en forma de roseta en los extremos de ramillas cortas originadas en años anteriores, algunas también más espaciadas sobre ramas jóvenes más alargadas, imparipinnadas, con (1-) 5-11 foliolos (las hojas con 1 a 3 foliolos son las primeras en aparecer en cada fascículo, por lo general son efímeras), raquis con frecuencia un poco ensanchado entre los foliolos superiores, al igual que el peciolo fina y esparcidamente pubérulo, pero glabrescente, foliolos sésiles o subsésiles, el terminal por lo general mucho más grande que los laterales, ovado a elíptico, romboide, oblanceolado u obovado, hasta de $3 \mathrm{~cm}$ de largo y $1.5 \mathrm{~cm}$ de ancho, ápice agudo a truncado, base cuneada, pero con cierta frecuencia asimétrica, debido a diversos grados de fusión con el foliolo lateral vecino, margen entero a irregular, foliolos laterales opuestos o subopuestos, por lo general elípticos a oblongos, hasta de $3 \mathrm{~cm}$ de largo y $1 \mathrm{~cm}$ de ancho, agudos a truncados y a veces emarginados en el ápice, asimétricamente cuneados en la base, margen entero, pero con frecuencia también irregular, pubescencia de los foliolos similar a la del resto de las partes de la planta.

Flores masculinas desconocidas y aparentemente ausentes. Flores femeninas apareciendo por lo general al mismo tiempo que las hojas, fasciculadas en los extremos de ramillas cortas, o bien, en la base de ramillas largas que están en desarrollo, solitarias o a veces en grupos de 2, pedúnculos hasta de $8 \mathrm{~mm}$ de largo, con algo de puberulencia fina y a menudo con una diminuta bráctea; lóbulos del cáliz 3 , triangulares, de 1 a $1.2 \mathrm{~mm}$ de largo, agudos en el ápice, ciliados en el margen; pétalos 3 , oblongos, de $\pm 3 \mathrm{~mm}$ de largo, de color crema-verdoso, glabros; estaminodios 6 , filamentos de poco menos de $0.5 \mathrm{~mm}$ de largo, anteras linearoblongas, de $\pm 1 \mathrm{~mm}$ de largo, dorsifijas; ovario trilocular, glabro, estigmas 3 . 


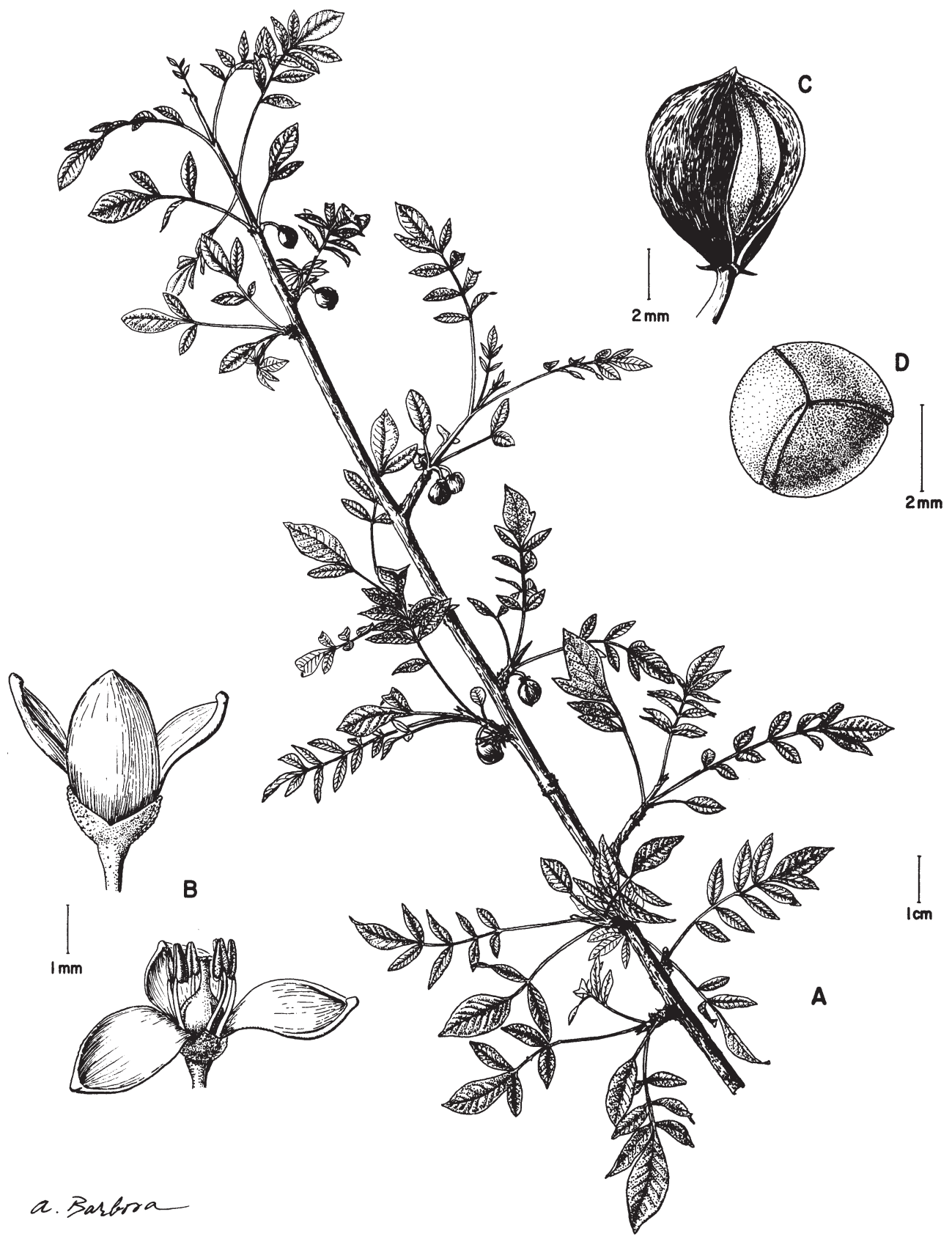

Fig. 1. Bursera medranoana Rzedowski \& Ortiz. A. Rama con frutos. B. Flores femeninas. C. Fruto con una de las valvas del mesocarpio removidas. D. Hueso cubierto de pseudoarilo. 
Drupas trivalvadas, subglobosas a obovoides, a menudo puntiagudas en el ápice, de 6 a $7 \mathrm{~mm}$ de largo, glabras, sobre pedúnculos engrosados y encorvados, hasta de $10 \mathrm{~mm}$ de largo; hueso subgloboso a obovoide, de 5 a $6 \mathrm{~mm}$ de largo, totalmente cubierto por el pseudoarilo que en la madurez es de color anaranjado-rojizo.

Florece en junio y pronto los frutos alcanzan su máximo tamaño, permaneciendo sobre el árbol por lo general durante muchos meses; las semillas no maduran simultáneamente.

El nombre de la especie se dedica como homenaje al M. en C. Francisco González Medrano, prestigiado botánico mexicano y activo promotor de los estudios de la flora y vegetación de las barrancas semiáridas del estado de Hidalgo. El Maestro Medrano herborizó los primeros ejemplares de este taxon.

TIPO: México, Hidalgo, fondo de la Barranca de Tolantongo, municipio de Cardonal, ladera caliza con vegetación de bosque de Bursera, alt. 1300 m, 30.V.1984, Rzedowski38401a (ENCB, holotipo) (hojas, flores femeninas y frutos tiernos); duplicados por distribuirse.

Material adicional examinado: México, Hidalgo, $1 \mathrm{~km}$ al E de la gruta, Tolantongo, mun. Cardonal, X.1976, F. G. Medrano et al. 10097 (ENCB) (hojas y frutos); Barranca de Tolantongo, cerca de las grutas, mpio. de Cardonal, 24. VIII.1980, Rzedowski36937(ENCB) (hojas y frutos); fondo de la Barranca de Tolantongo, mpio. de Cardonal, 29. IX.1982, Rzedowski37949(ENCB) (hojas y frutos); ibid., 16.X.1983, Rzedowski 38339. 38843 (ENCB) (hojas y frutos); ibid., 17.V.1984, Rzedowski 38396 (ENCB) (flores y hojas muy tiernas).

De los individuos de B. schlechtendalii $\times$ B. medranoana se herborizaron: México, Hidalgo; parte baja de la Barranca de Tolantongo, 19.Vl.1983, Rzedowski 38092 (ENCB); ibid., 16.X.1983, Rzedowski 38340 (ENCB).

Hasta ahora B. medranoana sólo se conoce de la localidad tipo y sería interesante explorar otros sitios vecinos en su búsqueda, como por ejemplo la cercana Barranca de Metztitlán, donde en algunos sitios se ha visto que crecen juntas $B$. morelensis y $B$. schlechtendalii. La convivencia de estas dos especies, por cierto, no se limita al estado de Hidalgo, sino que es frecuente también a lo largo de casi toda el área de distribución de B. morelensis.

\section{AGRADECIMIENTOS}

El dibujo que ilustra este artículo es obra de Alfonso Barbosa. Se agradece, además, al Biól. Rafael Fernández Nava su entusiasta colaboración en el trabajo de campo.

\section{LITERATURA CITADA}

Hiriart Valencia, P. 1981. Vegetación y fitogeografía de la Barranca de Tolantongo, Hidalgo, México. Tesis. Facultad de Ciencias. Universidad Nacional Autónoma de México. México, D.F. 98 pp.

McVaugh, R. y J. Rzedowski. 1965. Synopsis of the genus Bursera L. in western Mexico, with notes on the material of Bursera collected by Sessé \& Mociño. Kew Bull. 18: 317-382.

Rzedowski, J. 1970 (1968). Notas sobre el género Bursera (Burseraceae) en el estado de Guerrero. An. Esc. Nac. Cienc. Biol., Méx. 17: 17-36.

Rzedowski, J. y E. Ortiz. 1982. Estudios quimiotaxonómicos de Bursera (Burseraceae). I. Bursera chemapodicta sp.n. Bol. Soc. Bot. Méx. 43: 73-80. 